\section{Case Reports in Ophthalmology}

\title{
Catastrophic Thermal Corneoscleral Injury Treated with Transplantation of Donor Scleral Graft
}

\author{
Satoru Kase Takeshi Ohguchi Susumu Ishida \\ Department of Ophthalmology, Faculty of Medicine and Graduate School of Medicine, \\ Hokkaido University, Sapporo, Japan
}

\section{Keywords}

Thermal injury · Phacoemulsification · Donor sclera · Histopathology · Coagulation necrosis

\begin{abstract}
Background: The aim of this study is to report a patient with senile cataract developing severe thermal corneoscleral injury during phacoemulsification, which was treated with a donor scleral graft. Case: Severe thermal corneoscleral injury occurred during phacoemulsification in the right eye of a 74-year-old male. His medical history was prostate hypertrophy. Visual acuity was hand motion and the intraocular pressure was $3 \mathrm{~mm} \mathrm{Hg} O D$. There was heavy corneal stromal opacity with intraocular fluid leakage. The patient underwent transplantation of a donor scleral graft to the burn site. Histologically, the injured sclera showed coagulation necrosis without inflammatory cell infiltration. An intraocular lens was eventually fixed in the ciliary sulcus 7 months later. His visual acuity remains at 2/20 OD. Conclusions: Transplantation of the donor scleral grafts is useful to close the wound in catastrophic thermal injury.
\end{abstract}

(C) 2017 The Author(s)

Published by S. Karger AG, Basel

\section{Introduction}

Thermal corneoscleral injury is one of the serious complications during phacoemulsification in cataract surgeries. It has been considered that thermal injury may be caused by 
irrigation fluid disorder around the phacoemulsification tubes, which leads to difficulty of corneoscleral wound closure, wound leakage, and damage of the corneal endothelium [1]. Suturing of local burn sites, soft contact lens use, and amniotic membrane transplantation are standard therapeutic options to treat thermal corneoscleral injuries [2]. In this study, we report a patient with senile cataract developing severe thermal corneoscleral injury during phacoemulsification, which was treated with a donor scleral graft.

\section{Case Report}

A 74-year-old male complained of blurred vision in both eyes because of senile cataract in October 2015. Visual acuities were 6/20 OD and 8/20 OS with a normal intraocular pressure (IOP). Slit-lamp examination revealed mild cortical cataract OU. Fundus findings showed nothing of note. The initial ophthalmologist planned to perform cataract surgery for his right eye. His medical history was prostate hypertrophy, while he had no history of dementia. Viscoelastic materials with high-level cohesion were injected into the anterior chamber to dilate the pupil due to miosis following hydrodissection OD. Severe thermal corneoscleral injury occurred soon after beginning the phacoemulsification. The wound was tightly sutured by pedunculated conjunctiva; however, viscoelastic materials were injected again since the leakage was not suppressed. The next day, he was referred to our hospital. Visual acuity was hand motion and IOP was $3 \mathrm{~mm} \mathrm{Hg}$ OD. There was marked corneal stromal opacity (Fig. 1a) with intraocular fluid leakage. The scleral wound was found to be opening following conjunctival incision (Fig. 2a). Since mobility of the sclera was markedly involved, it was impossible to conduct direct suture of the injured sclera. Therefore, the patient underwent transplantation of a donor scleral graft using 10-0 nylon to the burn site (Fig. 2b). After the transplantation and confirmation of the absence of intraocular fluid leakage, the lens nucleus was extracted from the newly formed wound on the temporal side of the scald. Histologically, the injured sclera showed coagulation necrosis with eosinophilic materials (Fig. 2c). Attachment of the transplanted sclera was favorable and IOP recovered to normal. Three months after the surgery, macular edema occurred due to intraocular inflammation caused by the remaining lens cortex. Therefore, anterior vitrectomy was conducted leading to the resolution of macular edema, and an intraocular lens was eventually fixed in the ciliary sulcus 7 months later. The visual acuity improved to $2 / 20$ OD with stable IOP and a reduced corneal opacity in October 2016 (Fig. 1b).

\section{Discussion}

Donor scleral grafts are materials which support tissue defects associated with trabeculectomy, glaucoma tube shunt surgery, or enucleation [3]. Further, the scleral grafts have been used to reconstruct tissues in corneal perforation, and scleral thinning resulting from inflections or autoimmune diseases. In contrast, common treatments for thermal injury during cataract surgeries include suture of the sclera/conjunctiva, contact lens application, a fornix-based rectangular conjunctival flap, and amniotic membrane transplantation [2, 4]. Although these treatments could be useful in relatively mild thermal injury, severe scleral injury may be refractory since the scleral wound expands together with poor mobility of the sclera. This study demonstrates that transplantation of donor scleral grafts is useful to close the wound in severe thermal corneoscleral injury sites. 
Kase et al.: Catastrophic Thermal Corneoscleral Injury Treated with Transplantation of Donor Scleral Graft

The suspected cause of the catastrophic thermal burn in this case is related to (1) circulation disorder of irrigation fluid, (2) an excess amount of viscoelastic materials injected because of miosis following hydrodissection, and (3) the possible background of floppy iris syndrome associated with prostate hyperplasia. Moreover, this study, for the first time, demonstrated histological findings of characteristic drop-out of scleral stromal cells (Fig. 2c: asterisk) replaced by eosinophilic deposition within the injured sclera, which is consistent with coagulation necrosis. These pathological findings indicate irreversible changes in the collagen fibril structure observed in the thermally burned cornea [1]. In addition, no inflammatory cells infiltrated the scleral tissue, suggesting rapid irreversible destruction of the tissue by thermal injury.

\section{Conclusions}

The pathology of the sclera involved in thermal injury is coagulation necrosis. The transplantation of donor scleral grafts is useful to close the wound in catastrophic thermal injury.

\section{Statement of Ethics}

The authors have no ethical conflicts to disclose.

\section{Disclosure Statement}

None of the authors has any conflict of interest related to the publication of this paper.

\section{References}

1 Sippel KC, Pineda R Jr: Phacoemulsification and thermal wound injury. Semin Ophthalmol 2002;17:102-109.

-2 Shimazaki J, Yang HY, Tsubota K: Amniotic membrane transplantation for ocular surface reconstruction in patients with chemical and thermal burns. Ophthalmology 1997;104:2068-2076.

-3 Hodge C, Sutton G, Devasahayam R, Georges P, Treloggen J, Cooper S, Petsoglou C: The use of donor scleral patch in ophthalmic surgery. Cell Tissue Bank 2017;18:119-128.

4 Haldar K, Saraff R: Closure technique for leaking wound resulting from thermal injury during phacoemulsification. J Cataract Refract Surg 2014;40:1412-1414. 


\section{Case Reports in Ophthalmology}
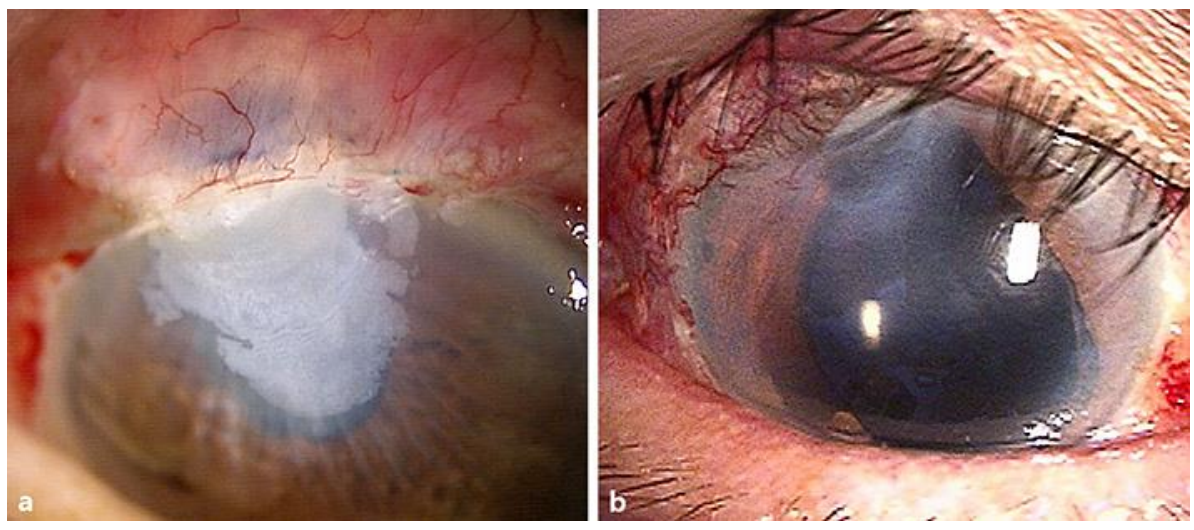

Fig. 1. Slit-lamp examination of the patient's right eye before (a) and 12 months after an initial surgery (b). There is marked corneal stromal opacity along with a marked Descemet membrane fold (a). Corneal opacity improves compared with the initial presentation with fine intraocular lens fixation (b).
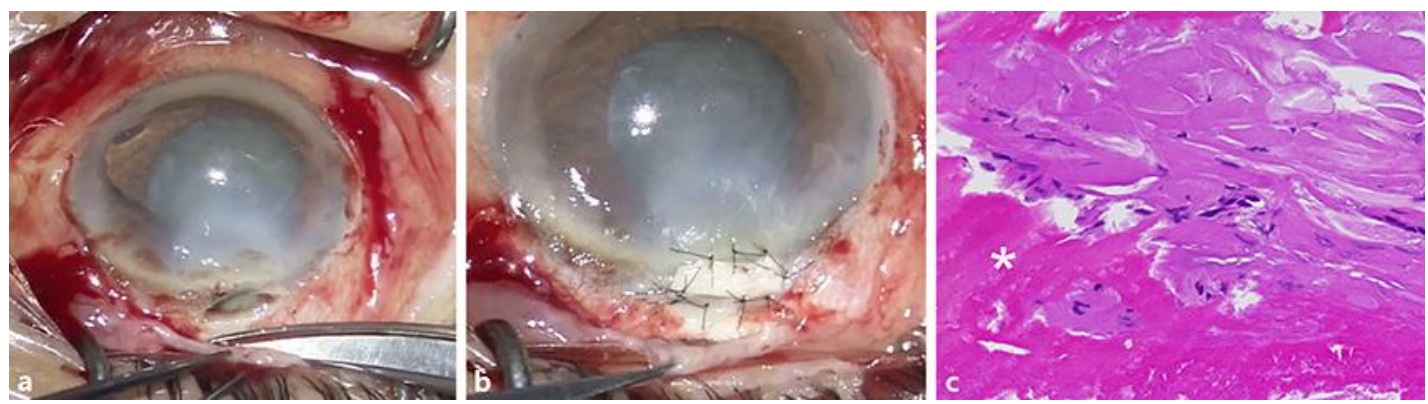

Fig. 2. Intraoperative microscopic findings in the patient's right eye (surgeon's view) (a, b) and histopathology of the thermally injured sclera (c). The scleral wound has completely opened following conjunctival incision (a). A donor scleral graft is transplanted using 10-0 nylon to the wound site (b). The normal scleral stroma is absent (asterisk) where eosinophilic materials are accumulated. No inflammatory cell infiltration is noted $(\mathbf{c})$. 